\title{
Robust Impulsive Stabilization of Uncertain Nonlinear Singular Systems with Application to Transportation Systems
}

\author{
Shaohong Fang $(\mathbb{D}$ \\ School of Software Engineering, Huazhong University of Science and Technology, Wuhan, Hubei 430074, China \\ Correspondence should be addressed to Shaohong Fang; fangshaohong@hust.edu.cn
}

Received 20 September 2018; Revised 13 November 2018; Accepted 27 November 2018; Published 6 December 2018

Guest Editor: Shenping Xiao

Copyright (C) 2018 Shaohong Fang. This is an open access article distributed under the Creative Commons Attribution License, which permits unrestricted use, distribution, and reproduction in any medium, provided the original work is properly cited.

\begin{abstract}
We consider the robust asymptotical stabilization problem for uncertain singular systems. We design a new impulsive control technique to ensure that the controlled singular system is robustly asymptotically stable and hence derive the corresponding stability criteria. These sufficient conditions are expressed in the form of algebra matrix inequalities and can be implemented numerically. We finally provide a numerical example of a transportation system to illustrate the effectiveness and usefulness of the proposed criteria.
\end{abstract}

\section{Introduction}

System stability is a fundamental issue for a nonlinear system. It not only relates to its system structure but also has relations with the exterior disturbance of the system. Singularity and parameter's uncertainty of the system can seriously affect its stability performance. For an unstable system, how to design a controller which stabilizes the system becomes a critical problem, which motivates the current research. The stabilization methods can be applied to a range of practical applications such as transportation systems $[1,2]$.

As for general nonlinear systems, various design methods to control these dynamical systems have been proposed. Among them, impulsive control methods have attracted considerable attention because impulsive control laws have a fast response time, strong robustness and resistance to disturbances, and low energy consumption. They have been applied to many disciplines [3-5]. The stabilization issue for the nonsingular certain system by use of the impulsive control method has been studied and many sufficient conditions of its asymptotical stability, such as [6-9], have been provided. Recent research work can be found in [10-15] and the references therein.
In this paper, we will develop the impulsive control method, the Lyapunov functional method, and the matrix inequality technology to solve the stabilizing issue of a class of singular systems with uncertainty, and design an impulsive controller of parts of state variables. Finally, the sufficient conditions of asymptotical stability will be given under our designed controller.

This paper's notations are quite standard. Let $R^{n}$ denote the n-dimensional Euclidean space, $R_{+}$the set of nonnegative real numbers, and $R^{m \times n}$ the set of all real $m \times n$ matrices. The superscript " $T$ " represents the matrix transposition operation and $X \geq Y$ (respectively, $X>Y$ ), where $X$ and $Y$ are symmetric matrices, indicates that $X-Y$ is positive semidefinite (respectively, positive definite). The symbol $I_{n}$ is $n \times n$ the identity matrix and $\|\cdot\|$ is the Euclidean norm in $R^{n}$. The $\lambda_{M}(P)$ and $\lambda_{m}(P)$ represent the largest and smallest eigenvalues of $P$, respectively. Let $K: R_{+} \longrightarrow R$ denote the set of all continuous real-valued functions. We say that the function $p: R_{+} \longrightarrow R_{+}$is piecewise continuous if it is continuous on $R_{+}$, except at the time points in the set $\left\{\tau_{k}\right\}$, is left-continuous, and has the right limit at $\tau_{k}$ for all $k$. The $P C\left(R_{+}, R_{+}\right)$is the set of all such piecewise continuous functions $p$. 


\section{Preliminaries}

2.1. Uncertain Singular System. Consider the following singular system with disturbances:

$$
\begin{aligned}
E \dot{x}(t) & =A x(t)+w(t), \\
y(t) & =C x(t), \\
x\left(t_{0}\right) & =x_{0},
\end{aligned}
$$

where $x(t) \in R^{n}$ and $y(t) \in R^{m}$ are the system state and output vectors, respectively. The parameter $w(t, x(t))$ is the norm-bounded external uncertainty described by a continuous vector-valued function. The matrices $A \in R^{n \times n}$ and $C \in R^{m \times n}$ are constant ones of appropriate dimensions. In the situation that $0<\operatorname{rank}(E)<n$, we say that system (1) is an uncertain singular system. Without loss of generality, we assume that

$$
E=\left(\begin{array}{cc}
I_{u} & 0 \\
0 & 0
\end{array}\right),
$$

where $I_{u} \in R^{u \times u}\left(u \in N^{+}, u<n\right)$ is an $u \times u$ identity matrix. We need the following assumptions for our later use.

Assumption 1. The uncertainty $w(t)$ is a norm-bounded nonlinear function and satisfies the following Lipschitz condition:

$$
\begin{aligned}
& \left\|w\left(t_{1}\right)-w\left(t_{2}\right)\right\| \leq l\left\|x_{u}\left(t_{1}\right)-x_{u}\left(t_{2}\right)\right\|, \\
& \forall t_{1}, t_{2} \in R_{+} \text {, }
\end{aligned}
$$

where $l>0$ is a known constant scalar.

2.2. Impulsive Control System Theory. Consider the following nonlinear system with impulses:

$$
\begin{aligned}
\dot{x}(t) & =f(t, x(t)), \quad t \neq \tau_{k}, \\
y & =\varphi(x), \quad t \neq \tau_{k}, \\
x\left(t_{0}^{+}\right) & =x_{0}, \quad k=1,2, \ldots, \\
\Delta x(t) & =u_{k}(y), \quad t=\tau_{k},
\end{aligned}
$$

where $0<\tau_{1}<\cdots<\tau_{k}<\cdots$, with $\tau_{k} \longrightarrow \infty$ as $k \longrightarrow \infty$, and

$$
\Delta x\left(\tau_{k}\right)=x\left(\tau_{k}^{+}\right)-x\left(\tau_{k}^{-}\right)=x\left(\tau_{k}^{+}\right)-x\left(\tau_{k}\right),
$$

with $x\left(\tau_{k}^{+}\right)=\lim _{t \rightarrow \tau_{k}^{+}} x(t)$, and $x\left(\tau_{k}^{-}\right)=\lim _{t \rightarrow \tau_{k}^{-}} x(t)$. Let $\varphi \in C\left(R^{n}, R^{m}\right), u_{k} \in C\left(R^{m}, R^{n}\right), f \in C\left(R_{+} \times R^{n}, R^{n}\right)$. The $C\left(R^{n}, R^{m}\right)$ is defined to be the set of all continuous functions mapping the value in $R^{n}$ to the value in $R^{m}$ and $C\left(R^{m}, R^{n}\right)$ and $C\left(R_{+} \times R^{n}, R^{n}\right)$ can be defined similarly.

Definition 2. A sequence pair $\left\{\tau_{k}, u_{k}\left(y\left(\tau_{k}\right)\right)\right\}$ is said to be an impulsive control pair for system (4a)-(4c). If system (4a)(4c) is asymptotically stable after implementing an impulsive control, system (4a)-(4c) is said to be robustly impulsively stabilizable.
Given $\rho>0$, let $S_{\rho}=\left\{x \in R^{n}:\|x(t)\|<\rho\right\}$. For $k=$ $1,2, \ldots$ and $(t, x) \in\left(\tau_{k-1}, \tau_{k}\right] \times R^{n}$, define

$$
\begin{aligned}
& D^{+} V(t, x) \\
& \quad=\lim _{h \rightarrow 0} \sup \frac{1}{h}[V(t+h, x+h f(t, x))-V(t, x)] .
\end{aligned}
$$

Furthermore, let $V(\cdot, \cdot): R_{+} \times S_{\rho} \longrightarrow R_{+}$be a continuous function on $R_{+} \times S_{\rho}$, except possibly at the time points $\left\{\tau_{k}\right\}$ satisfying the following two conditions:

(1) For each $x \in S_{\rho}, k=1,2, \ldots, \lim _{(t, y) \longrightarrow\left(\tau_{k}^{-}, x\right)} V(t, y)=$ $V\left(\tau_{k}^{-}, x\right)$ exists;

(2) $V(t, x)$ is locally Lipschitz in $x$.

To proceed, we need the following lemmas.

Lemma 3 (see [7]). Let $\varphi\left(0^{-}\right)=\varphi(0)$ and $\tau=\sup _{k}\left\{\tau_{k+1}-\right.$ $\left.\tau_{k}\right\}<\infty$. Suppose that there exist $\alpha, \beta, c, \psi \in K, p \in$ $P C\left(R_{+}, R_{+}\right), V(t, x) \in V_{0}$, and $\sigma>0$ such that

(1) $x+u_{k}(x) \in S_{\rho}$ for all $k$;

(2) $\beta(\|x\|) \leq V(t, x) \leq \alpha(\|x\|), \forall(t, x) \in R_{+} \times S_{\rho}$;

(3) $V\left(\tau_{k}, x+u_{k}(x)\right) \leq \psi_{k}\left(V\left(\tau_{k}, x\right)\right), k=1,2, \ldots$;

(4) $D^{+} V(t, x) \leq p(t) c(V(t, x))$, for $t \neq \tau_{k}$ and $x \in S_{\rho}$;

(5) $\int_{q}^{\psi_{k}(q)}(d s / c(s))+\int_{\tau_{k}}^{\tau_{k+1}} p(s) d s \leq-l_{k}, \forall q \in(0, \sigma)$, for all $l_{k} \in(0,+\infty), k=1,2, \ldots$, and $\sum_{k=1}^{\infty} l_{k}=\infty$.

Then, system (4a), (4b), (4c), and (4d) is asymptotically stable.

Lemma 4 (see [8]). Given a positive matrix $S$ and a positive scalar $\gamma$, we have

$$
M N+N^{T} M^{T} \leq \gamma^{-1} M S^{-1} M^{T}+\gamma N^{T} S N,
$$

where $M$ and $N$ are real matrices with appropriate dimensions.

\section{Robust Impulsive Stabilization}

In this section, we shall design an impulsive control pair $\left\{\tau_{k}, u_{k}\left(x_{u}\right)=B x_{u}\left(\tau_{k}\right)\right\}$ to stabilize system (1). System (1) under impulsive control can be rewritten as

$$
\begin{aligned}
\dot{x}_{u}(t) & =A_{11} x_{u}(t)+A_{12} x_{v}(t)+w_{u}(t), \quad t \neq \tau_{k}, \\
\Delta x_{u}(t) & =x_{u}\left(t^{+}\right)-x_{u}\left(t^{-}\right)=B x_{u}(t), \quad t=\tau_{k}, \\
0 & =A_{21} x_{u}(t)+A_{22} x_{v}(t)+w_{v}(t), \quad t \neq \tau_{k}, \\
y(t) & =C x(t),
\end{aligned}
$$

where $x(t)=\left(x_{u}(t), x_{v}(t)\right)^{T}, w(t)=\left(w_{u}(t), w_{v}(t)\right)^{T}$ and $B \epsilon$ $R^{u \times u}$. The initial conditions are given as $x_{u}\left(t_{0}\right)=x_{u 0}$, and $x_{v}\left(t_{0}\right)=x_{v 0}, \tau_{k} \geq t_{0}, k=1,2, \cdots$.

Theorem 5. Suppose Assumption 1 holds. The impulsive controlled system ( $8 a),(8 b),(8 c)$, and ( $8 d)$ is robustly asymptotically stable if the following conditions are satisfied: 
(1) $\lambda_{M}\left(Q_{2}\right)<1$;

(2) $\alpha=\lambda_{m}\left(A_{22}^{T} A_{22}\right)>0$;

(3) $\lambda_{M}\left(Q_{1}\right) \tau+\ln \lambda_{M}\left(Q_{2}\right)<0$.

where

$$
\begin{aligned}
Q_{1}= & A_{11}+A_{11}^{T}+A_{12} A_{12}^{T}+\frac{1}{\alpha} A_{21}^{T} A_{21} \\
& +\left(1+m l^{2}\right) I_{p}, \\
Q_{2}= & \left(I_{p}+B\right)^{T}\left(I_{p}+B\right) \\
\text { and } m= & \max \left\{1, \frac{2}{\alpha}\right\} .
\end{aligned}
$$

Proof. Consider the following standard quadratic Lyapunov function candidate:

$$
V(t, x)=x_{u}(t)^{T} x_{u}(t),
$$

which satisfies condition (i) of Lemma 3 . By virtue of the upper Dini derivative of Lyapunov function (10) along the solution of system $(8 \mathrm{a}),(8 \mathrm{~b}),(8 \mathrm{c})$, and $(8 \mathrm{~d})$, it follows that at $t \neq \tau_{k}, k=1,2, \ldots$, it yields

$$
\begin{aligned}
D^{+} V(t, x)= & \dot{x}_{u}(t)^{T} x_{u}(t)+x_{u}(t)^{T} \dot{x}_{u}(t) \\
= & x_{u}(t)^{T}\left(A_{11}+A_{11}^{T}\right) x_{u}(t) \\
& +2 x_{u}(t)^{T} A_{12} x_{v}+2 x_{u}(t)^{T} w_{u}(t) .
\end{aligned}
$$

By Lemma 4, the following inequalities

$$
2 x_{u}(t)^{T} A_{12} x_{v} \leq x_{u}(t)^{T} A_{12} A_{12}^{T} x_{u}(t)+x_{v}^{T} x_{v},
$$

and

$$
2 x_{u}(t)^{T} w_{u}(t) \leq x_{u}(t)^{T} x_{u}(t)+w_{u}(t)^{T} w_{u}(t)
$$

hold. Thus, it follows that (11) can be rewritten as

$$
\begin{aligned}
D^{+} V(t, x) \leq & x_{v}(t)^{T} x_{v}+w_{u}(t)^{T} w_{u}(t) \\
& +x_{u}(t)^{T}\left(A_{11}+A_{11}^{T}\right) x_{u}(t) \\
& +x_{u}(t)^{T}\left(I_{u}+A_{12}^{T} A_{12}\right) x_{u}(t) .
\end{aligned}
$$

Moreover, we have

$$
\begin{aligned}
\lambda_{m}\left(A_{22}^{T} A_{22}\right) x_{v}^{T} x_{v} & \leq\left(A_{22} x_{v}\right)^{T}\left(A_{22} x_{v}\right) \\
& \leq 3 x_{u}^{T} A_{21}^{T} A_{21} x_{u}+3 w_{v}^{T} w_{v} .
\end{aligned}
$$

Using condition (4a), (4b), (4c), and (4d) of Theorem 5, we obtain

$$
x_{v}^{T} x_{v} \leq \frac{3}{\alpha} x_{u}^{T} A_{21}^{T} A_{21} x_{u}+\frac{3}{\alpha} w_{v}^{T} w_{v}
$$

and

$$
\frac{3}{\alpha} w_{v}^{T} w_{v}+w_{u}^{T} w_{u} \leq m l^{2} x_{u}^{T} x_{u}
$$

Combining (14), (16), and (17) together, we have

$$
D^{+} V(t, x) \leq \lambda_{M}\left(Q_{1}\right) V(t) .
$$

Thus, condition (8a), (8b), (8c), (8d) is satisfied with $p(s)=$ $\lambda_{M}\left(Q_{1}\right)$ and $c(s)=s$.

$$
\text { At } t=\tau_{k}, k=1,2, \ldots \text {, we have }
$$

$$
\begin{aligned}
V\left(x_{u}(t)+B x_{u}(t)\right) & =x_{u}^{T}\left(I_{u}+B\right)^{T}\left(I_{u}+B\right) x_{u} \\
& \leq \lambda_{M}\left(Q_{2}\right) V\left(x_{u}(t)\right) .
\end{aligned}
$$

Then condition (7) of Lemma 3 is satisfied with $\psi_{k}(s)=$ $\lambda_{M}\left(Q_{2}\right) s$. It follows from condition (1) of Theorem 5 and (19) that

$$
\left\|x_{u}(t)+B x_{u}(t)\right\| \leq\left\|x_{u}\right\|,
$$

which indicates that $x_{u} \in S_{\rho_{0}}, \rho_{0} \in(0, \rho)$; i.e., $x_{u}+B x_{u} \in S_{\rho_{0}}$ for all $k$. Thus, condition (ii) of Lemma 3 is also guaranteed. For $\tau<\infty$, the following two integrals are valid:

$$
\int_{q}^{\psi_{k}(q)} \frac{d s}{c(s)}=\ln \lambda_{M}\left(Q_{2}\right)
$$

and

$$
\int_{\tau_{k}}^{\tau_{k+1}} p(s) d s \leq \lambda_{M}\left(Q_{1}\right)\left(\tau_{k+1}-\tau_{k}\right) .
$$

Hence, it follows from condition (7) of Theorem 5 that

$$
\begin{aligned}
& \int_{q}^{\varphi_{k}(q)} \frac{d s}{c(s)}+\int_{\tau_{k}}^{\tau_{k+1}} p(s) d s \leq \lambda_{M}\left(Q_{1}\right) \tau+\ln \lambda_{M}\left(Q_{2}\right) \\
& \quad=-r_{k}<0
\end{aligned}
$$

and

$$
\sum_{k=1}^{\infty} l_{k}=\infty
$$

Condition (10) of Lemma 3 is also satisfied. Therefore, by Lemma 3 and Definition 2, system (8a)-(8b) is robustly asymptotically stable. Obviously, we can see that $\lim _{t \rightarrow+\infty} x_{u}(t)=0$ as $\|w(t)\| \leq l\left\|x_{u}(t)\right\|$, which leads to $\lim _{t \rightarrow+\infty} x(t)=0$. Therefore, system (8a), (8b), (8c), and (8c) is robustly asymptotically stable under the designed impulsive control. The proof is complete.

\section{Application to Transportation Systems}

In this section, we will consider a transportation application to illustrate our results obtained in Section 3. Consider a 
transportation system which is modelled by (1) with the following specifications:

$$
\begin{aligned}
A_{11} & =1, \\
A_{12} & =0.52, \\
A_{21} & =-0.48 \\
A_{22} & =2 \\
w(t) & =\left(\sqrt{\frac{x_{1}^{2}}{2}+x_{2}}, \sqrt{\frac{x_{1}^{2}}{2}-x_{2}}\right)^{T}, \\
E & =\left(\begin{array}{ll}
1 & 0 \\
0 & 0
\end{array}\right), \\
x(0) & =(-2,1)^{T} .
\end{aligned}
$$

It is clear that $l=1$. Then this transportation system is a singular 2-dimensional system with uncertainty. Now, we design an impulsive pair $\left\{\tau_{k}, B x_{1}\left(\tau_{k}\right)\right\}$, where $B=-0.8$ and $\tau=\tau_{k+1}-\tau_{k}=0.02$. Then, we have $\alpha=1>0, Q_{1}=18$, $Q_{2}=0.04$, and $\lambda_{M}\left(Q_{1}\right) \tau+\ln \lambda_{M}\left(Q_{2}\right)=-3<0$.

Consequently, it follows from Theorem 5 that the transportation system is impulsively stabilizable under the following impulsive control pair:

$$
\left\{\tau_{k}, B x_{1}\left(\tau_{k}\right)=-0.8 x_{1}\left(\tau_{k}\right)\right\}
$$

From the example, it is concluded that the impulsive control method can effectively stabilize the transportation singular systems.

\section{Conclusion}

In this paper, we have proposed a design method for robust impulsive stabilizing control for a singular transportation system with uncertainty. Sufficient conditions are derived to guarantee the global asymptotical stability of the system. An application to transportation systems shows that our designed impulsive stabilizing control is effective and strongly robust.

\section{Data Availability}

The data used to support the findings of this study are available from the corresponding author upon request.

\section{Conflicts of Interest}

The author declares that they have no conflicts of interest.

\section{Acknowledgments}

This work is supported by the National Natural Science Foundation of China (61873100).

\section{References}

[1] H. X. Ge, S. Q. Dai, L. Y. Dong, and Y. Xue, "Stabilization effect of traffic flow in an extended car-following model based on an intelligent transportation system application," Physical Review E, vol. 70, no. 6, Article ID 066134, 2004.

[2] A. S. Nair, J.-C. Liu, L. Rilett, and S. Gupta, "Non-linear analysis of traffic flow," in Proceedings of the 2001 IEEE Intelligent Transportation Systems Proceedings, pp. 681-685, August 2001.

[3] R. Z. Luo, "Impulsive control and synchronization of a new chaotic system," Acta Physica Sinica, vol. 56, no. 10, pp. 5655$5660,2007$.

[4] X. Liu and K. L. Teo, "Impulsive control of chaotic system," International Journal of Bifurcation and Chaos, vol. 12, no. 5, pp. 1181-1190, 2002.

[5] T. Yang, L. B. Yang, and C. M. Yang, "Impulsive control of Lorenz system," Physica D: Nonlinear Phenomena, vol. 110, no. 1-2, pp. 18-24, 1997.

[6] T. Yang, "Impulsive control," IEEE Transactions on Automatic Control, vol. 44, no. 5, pp. 1081-1083, 1999.

[7] X. Liu and G. Ballinger, "Uniform asymptotic stability of impulsive delay differential equations," Computers \& Mathematics with Applications, vol. 41, no. 7-8, pp. 903-915, 2001.

[8] Y.-Y. Cao, Y.-X. Sun, and C. Cheng, "Delay-dependent robust stabilization of uncertain systems with multiple state delays," IEEE Transactions on Automatic Control, vol. 43, no. 11, pp. $1608-1612,1998$.

[9] X. Xie, H. Xu, and R. Zhang, "Exponential stabilization of impulsive switched systems with time delays using guaranteed cost control," Abstract and Applied Analysis, vol. 2014, Article ID 126836, 8 pages, 2014.

[10] H. Xu, X. Liu, and K. L. Teo, "Robust $\mathrm{H}_{\infty}$ stabilisation with definite attenuance of an uncertain impulsive switche system," The ANZIAM Journal, vol. 46, no. 4, pp. 471-484, 2005.

[11] H. Xu, Y. Chen, and K. L. Teo, "Global exponential stability of impulsive discrete-time neural networks with time-varying delays," Applied Mathematics and Computation, vol. 217, no. 2, pp. 537-544, 2010.

[12] C. Briat, "Dwell-time stability and stabilization conditions for linear positive impulsive and switched systems," Nonlinear Analysis: Hybrid Systems, vol. 24, pp. 198-226, 2017.

[13] Y. Chen and H. Xu, "Exponential stability analysis and impulsive tracking control of uncertain time-delayed systems," Journal of Global Optimization, vol. 52, no. 2, pp. 323-334, 2012.

[14] H. Xu and K. L. Teo, "Ho optimal stabilization of a class of uncertain impulsive systems: An LMI approach," Journal of Industrial and Management Optimization, vol. 5, no. 1, pp. 153159, 2009.

[15] K. H. Degue, D. Efimov, and J.-P. Richard, "Stabilization of linear impulsive systems under dwell-time constraints: Interval observer-based framework," European Journal of Control, vol. 42, pp. 1-14, 2018. 


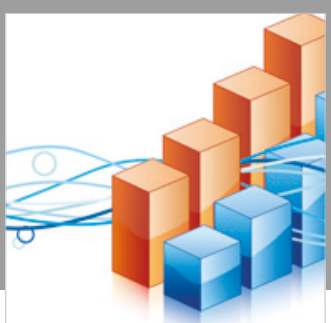

Advances in

Operations Research

\section{-n-m}
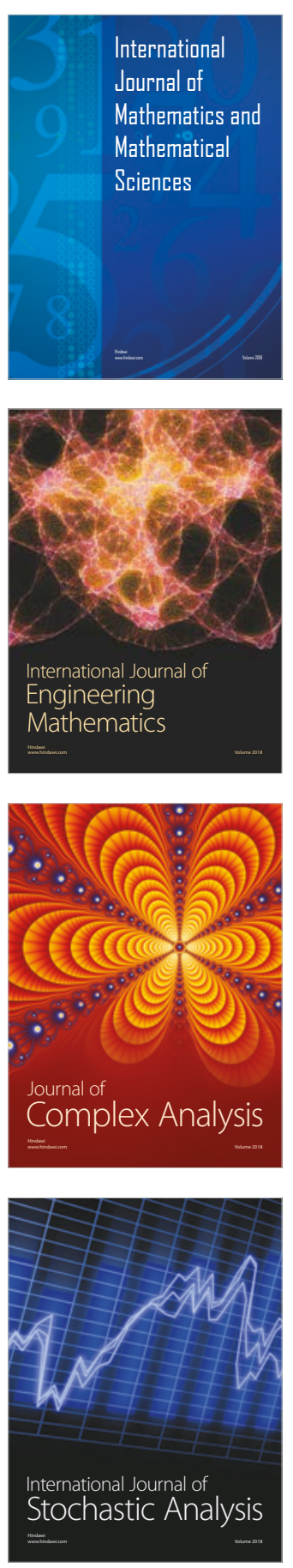
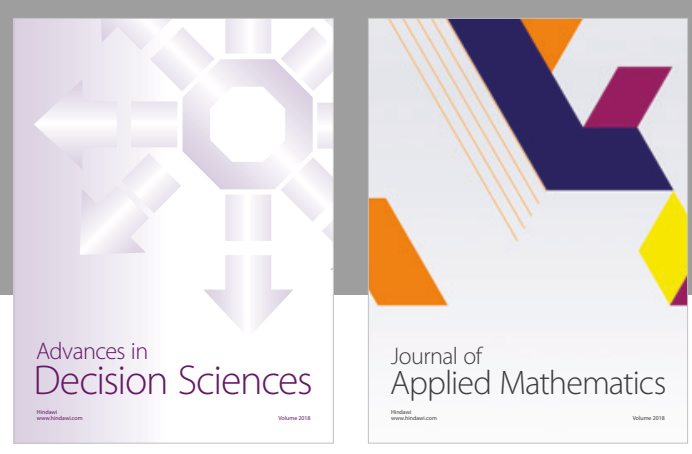

Journal of

Applied Mathematics
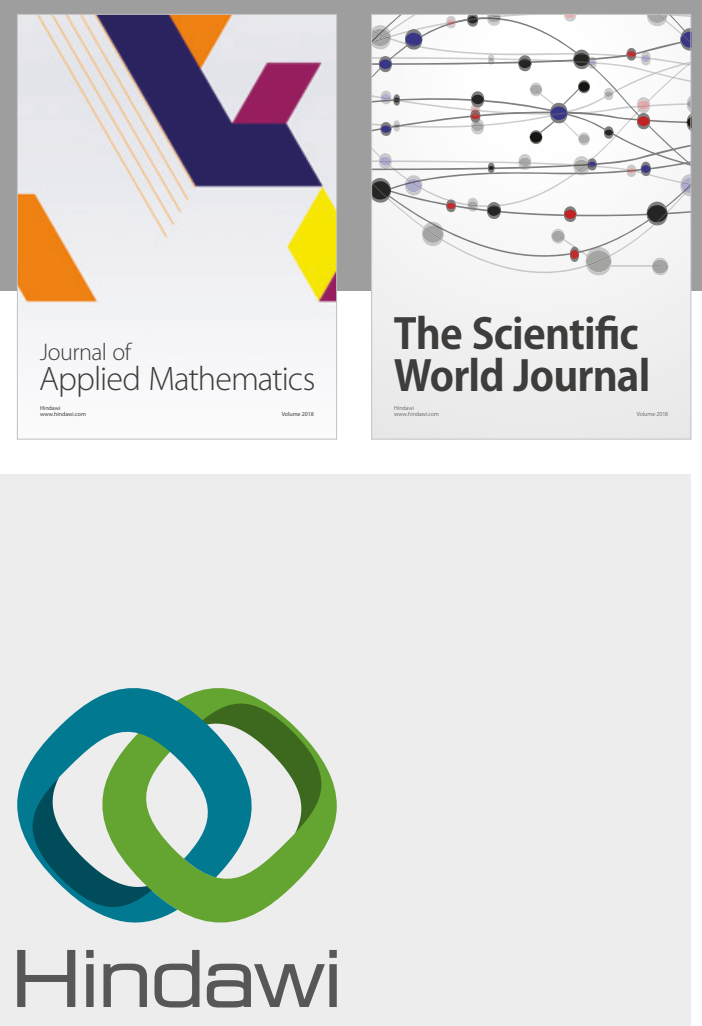

Submit your manuscripts at

www.hindawi.com

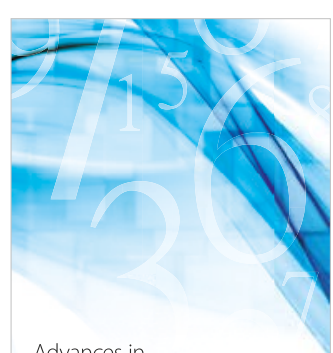

Advances in
Numerical Analysis
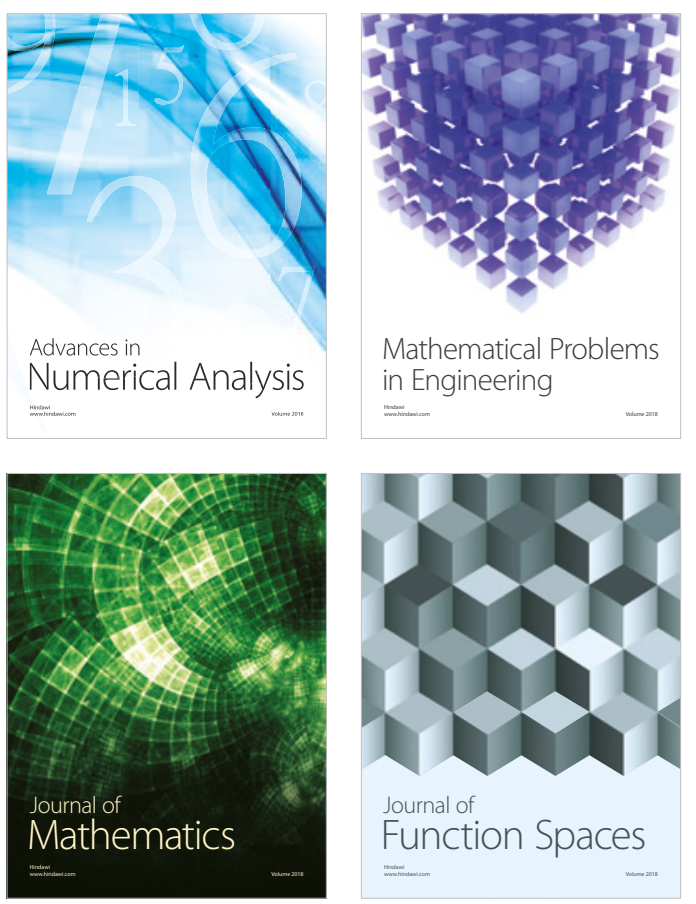

Mathematical Problems in Engineering

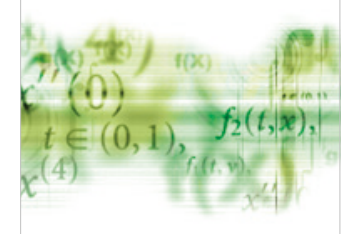

International Journal of

Differential Equations

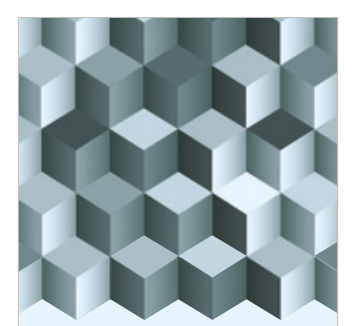

Journal of

Function Spaces

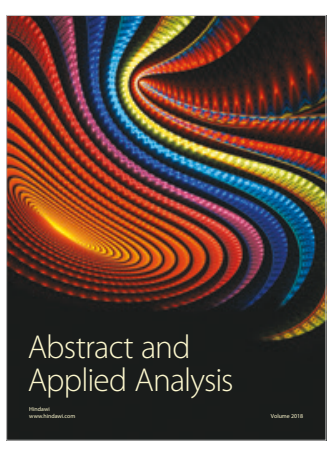

The Scientific

World Journal

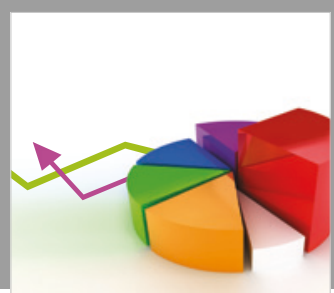

Journal of

Probability and Statistics
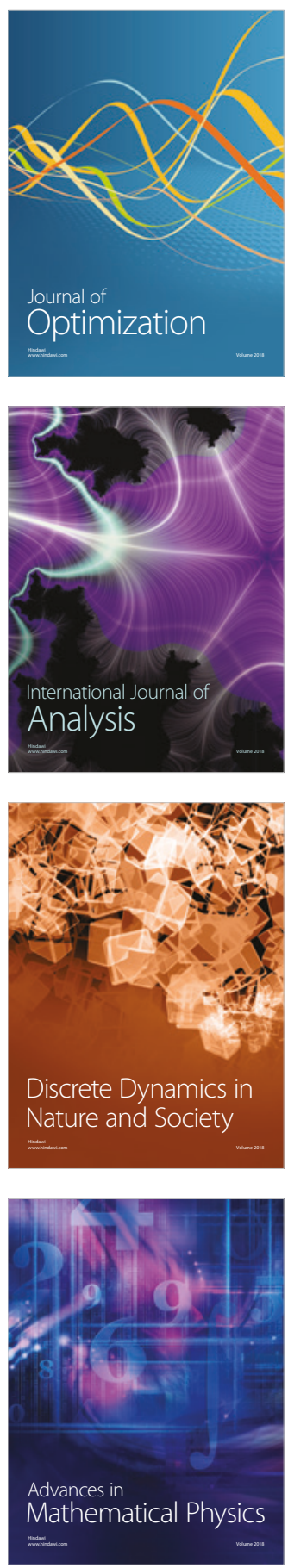